\title{
Pulmonary Vein Vasculitis Presenting as Multiple Pulmonary Nodules in a Patient with Systemic Lupus Erythematosus
}

HIROYA TAMAI@, MD; NAOSHI NISHINA @ , MD, PhD; TSUTOMU TAKEUCHI@, MD, PhD, Keio University School of Medicine, Tokyo, Japan. Address correspondence to Dr. H. Tamai, 35 Shinano-machi, Shinjuku-ku, Tokyo, Japan, 160-8582. E-mail: h.tamai@keio.jp. Ethics board approval is not required because this is a single case report and no intervention had been made for research. The patient gave written informed consent to publish the material. J Rheumatol 2019;46:323-4; doi:10.3899/jrheum.180602

Pulmonary involvement in systemic lupus erythematosus (SLE) can take the form of pleuritis, interstitial lung disease, alveolar hemorrhage, or pulmonary hypertension, but rarely does it appear as pulmonary vein vasculitis ${ }^{1}$.

A 19-year-old woman was diagnosed with SLE 6 months before presentation because of malar rash, alopecia, arthritis, leukocytopenia, low complement, and positive anti-DNA antibody, and $30 \mathrm{mg}$ daily prednisolone (PSL) treatment was started. Fever, skin ulcers on the scalp, and dry cough appeared 2 months before presentation, while she was taking $15 \mathrm{mg}$ of PSL. Thoracic computed tomography (CT) scan revealed bilateral multiple nodules distributed along pulmonary veins (Figure 1A).

The patient had jaundice and discoid lesions with ulcers on the scalp on physical examination. Laboratory findings showed acute liver dysfunction, decreased complement titer, positive anti-DNA antibody, negative antiphospholipid antibody, and no kidney involvement. Lung biopsy revealed lymphocytes and foam cell infiltration and fibrosis around pulmonary veins (Figure 2C-E) with disrupted internal elastic lamina (Figure 2F). Methylprednisolone pulse therapy followed by $50 \mathrm{mg}$ of daily PSL was started, and intravenous cyclophosphamide treatment was added. The discoid lesions resolved with scarring, and liver dysfunction improved soon after the initiation of therapy. One month later, thoracic CT scan showed that the pulmonary nodules were drastically reduced in size (Figure 1B).

Our original view was that the patient's pulmonary vein vasculitis was a manifestation of SLE because the SLE-specific discoid lesions were exacerbated at the same time. Although we sometimes see SLE with vasculitis, this case included the extremely unusual presentation of vasculitis limited to pulmonary veins ${ }^{2}$. This case highlights the possibility of vasculitis when nodules along pulmonary veins are found.

\section{ACKNOWLEDGMENT}

We thank Dr. H. Sugiura for his advice on radiological findings. We also thank Dr. A. Sasaki and Dr. K. Kameyama for useful comments on the pathology.

\section{REFERENCES}

1. Mittoo S, Fell CD. Pulmonary manifestations of systemic lupus erythematosus. Semin Respir Crit Care Med 2014;35:249-54.

2. Ramos-Casals M, Nardi N, Lagrutta M, Brito-Zeron P, Bove A, Delgado G, et al. Vasculitis in systemic lupus erythematosus: Prevalence and clinical characteristics in 670 patients. Medicine 2006;85:95-104.

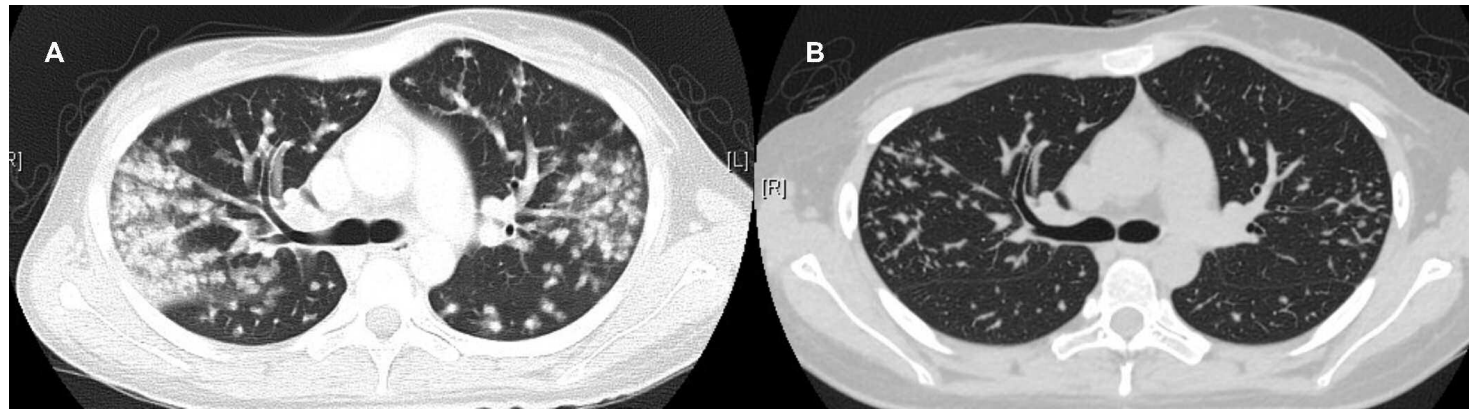

Figure 1. CT manifestations. A. CT scan revealed nodules distributed along pulmonary veins. B. CT scan taken 1 month later showed that the pulmonary nodules were drastically reduced in size. CT: computed tomography. 


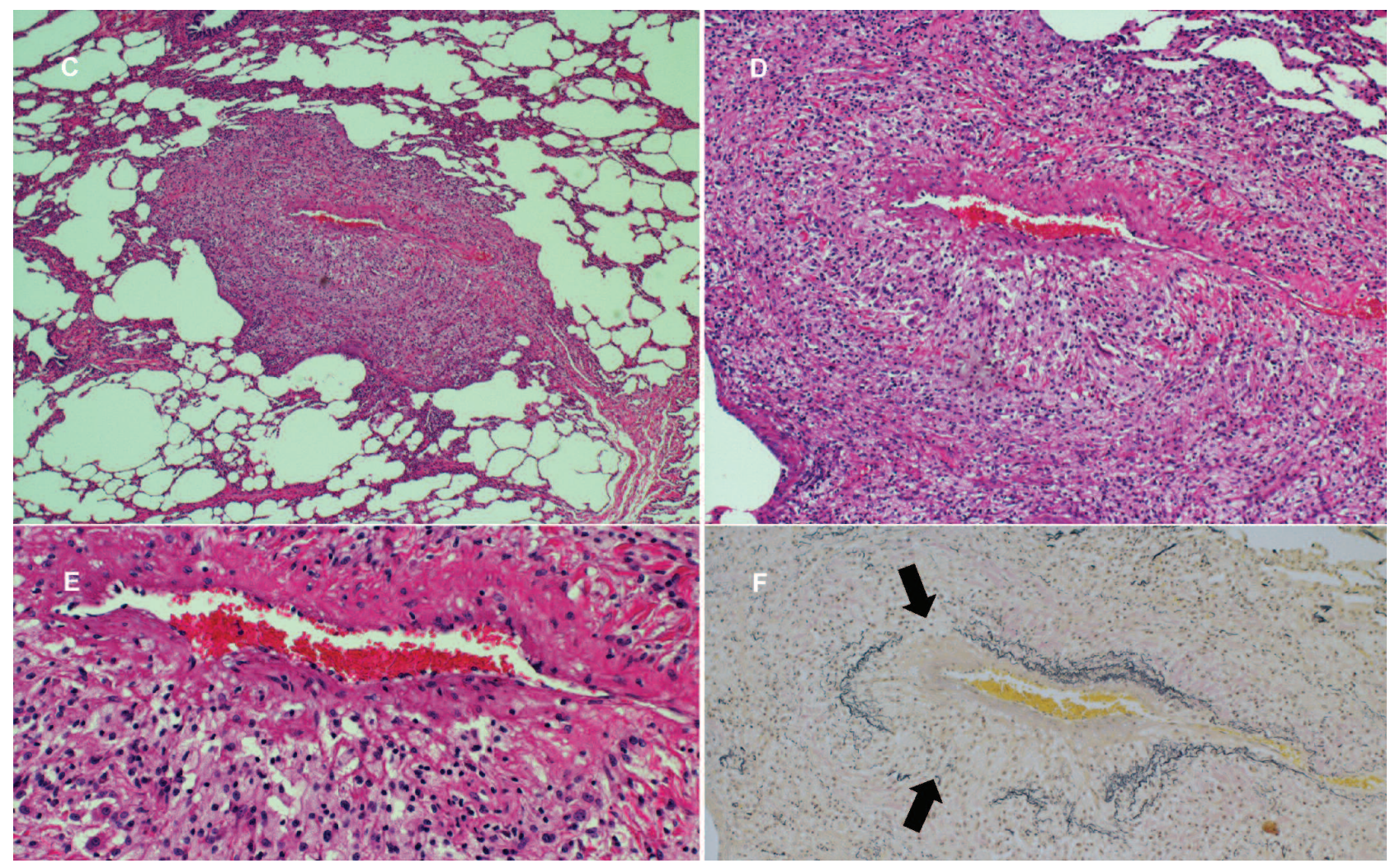

Figure 2. Histopathology of the lung tissue. Panels C-E show lung biopsy that revealed lymphocytes and foam cell infiltration and fibrosis around pulmonary veins, but without pulmonary artery lesions or thrombosis (H\&E staining, magnification C: $\times 40$, D: $\times 100$, E: $\times 200)$. F. Disrupted internal elastic lamina were also seen (arrows, Elastica van Gieson staining, magnification $\times 100$ ). 